\title{
Different ancestor alleles: a reason for the bimodal fragment size distribution in the minisatellite D2S44 (YNH24)
}

\author{
G Holmlund and B Lindblom \\ National Board of Forensic Medicine, Institute of Forensic Genetics, Linköping, Sweden
}

\begin{abstract}
Three conserved repeat order motifs found in the D2S44 (YNH24) minisatellite by minisatellite variant repeat mapping by PCR (MVR-PCR) might be the remnants of three different ancestor alleles. One of the motifs is found in the group of alleles around the $2.8 \mathrm{kbp}$ fragment size and the two others in the group of alleles around the $4.1 \mathrm{kbp}$ fragment size in the bimodal frequency distribution of Hinfl fragments characteristic for the D2S44 minisatellite. The conserved repeat-order motifs were found at the 3 '-end of the alleles, whilst the 5'-end was highly polymorphic. This is the first time a minisatellite is shown to have repeat-order motifs that are associated with fragment length. These results were obtained by MVR-PCR mapping of 49 alleles with primers specific for the core nucleotide sequence variation ...(G) $)_{5}$ TGTT, ...(G) $)_{4}$ TTGTT or ...(G) ${ }_{4}$ TGTT.
\end{abstract}

Keywords: minisatellite repeats; evolution

\section{Introduction}

The D2S44 (YNH24) minisatellite ${ }^{1}$ is located at 2q21.3-q22 border $^{2}$ close to the centromere on the long arm of the human chromosome 2 . This location is not far from the fusion region at $2 \mathrm{q}_{13} 3^{3}$ of two ancestor primate chromosomes. ${ }^{4}$ Population analysis of the D2S44 restriction fragment length polymorphism reveals a characteristic bimodal fragment size distribution, particularly in Caucasian populations. Cleaved with HinfI the two most frequent fragment sizes are 2.8 and $4.1 \mathrm{kbp}$. A bimodal fragment distribution is obtained with various enzymes and can therefore not be caused by restriction site polymorphism, but must

Correspondence: Gunilla Holmlund, National Board of Forensic Medicine, Institute of Forensic Genetics, University Hospital, S-581 85 Linköping, Sweden. Tel: + 46132230 36; Fax: + 46131360 05; E-mail: gunilla.holmlund@rs.rmv.se Received 30 December 1997; revised 22 April 1998; accepted 9 May 1998 rather be due to differences between the alleles in the two size groups. Detailed studies of individual minisatellite alleles have been undertaken using PCRprimers that recognise minute variations in the core sequences. $^{5-9}$ Such an analysis named minisatellite variant repeat mapping by PCR (MVR-PCR) can accordingly be used to reveal the internal order of different repeats in individual alleles. ${ }^{6}$ In most minisatellites the variation in the internal repeat order is focused at one end of the repeat array, MS31 (D7S21) ${ }^{10}$, MS32 (D1S8) ${ }^{5,6}$ and MS205 (D16S309), ${ }^{7}$ so called polarisation. However, both the CEB1 (D2S90) ${ }^{9}$ and MS621 (D5S110) ${ }^{11}$ minisatellites show no such polarisation. By MVR- mapping it has been established that most de novo mutations are found at the highly polymorphic end of the repeat array. MVR-PCR mapping has extensively been used for detailed studies of minisatellite mutation mechanisms. These studies include spontaneous mutations, ${ }^{12}$ mutations induced by 
radiation $^{13}$ as well as mutations in minisatellites inserted into yeast chromosomes. ${ }^{14}$ MVR-PCR analysis of the minisatellite MS205 in population studies has also been used to support further the human African origin. $^{8}$ We have earlier shown that the $2 \mathrm{kbp}$ D2S44 sequence in pYNH24 consists of a central array of repeated core sequences flanked at the $5^{\prime}$ end by a nucleotide sequence with degenerate repeats whilst the $3^{\prime}$ end contains a nonrepetitive unique nucleotide sequence. ${ }^{15}$ This was shown by a partial digestion with the restriction enzyme $R s a$ I that cleaves once in every core sequence. Partial digestion analysis revealed only the number of repeats, which is too little information for comparison of individual alleles. To get further information, we therefore determined the nucleotide sequence variation in the $31 \mathrm{bp}$ core sequence published earlier ${ }^{1}$ by cloning and sequencing a $2.25 \mathrm{kbp}$ HinfI allele, containing 14 consecutive RsaI sites. Variation in the core sequence is the prerequisite for an MVR-PCR analysis.

The frequency distribution of the D2S44 fragments into two groups suggests that the polymorphism of this minisatellite could originate from a duplication or a deletion of a certain section of the repeat array. It has been shown that the distribution of fragments into these two groups can vary between populations. For example the frequency of fragments between 2.7 and $2.9 \mathrm{kbp}$ is in Caucasians 0.119 and the frequency of fragments between 4.0 and $4.3 \mathrm{kbp}$ is 0.156 , whilst in US Blacks these frequencies are 0.065 and 0.051 respectively. ${ }^{16}$ According to this the duplication/deletion must have arisen early in human evolution or the two size groups might originate from different ancestor alleles. To compare the alleles belonging to the $2.8 \mathrm{kbp}$ group with those belonging to the $4.1 \mathrm{kbp}$ group we have analysed 49 D2S44 alleles, including pYNH24, from a Caucasian population by MVR-PCR.

\section{Materials and Methods}

Sequence Analysis of a $2.25 \mathrm{kbp}$ Hinf/ D2S44 Allele To determine the nucleotide variation in the D2S44 core sequence a $2.25 \mathrm{kbp}$ allele was amplified using the YNH24 specific primers described earlier. ${ }^{15}$ A PstI site was introduced into a tail of the 3 '- primer to perform a directional forced ligation of fragments of different lengths. The amplified allele was digested with PstI, partially with $R s a \mathrm{I}$ and ligated into the phagemid $\mathrm{SK}^{+}$(Stratagene) cleaved with PstI and HindII. The ligates were transformed into the E. coli strain XL1-Blue (Stratagene). Clones were selected by PCR using a YNH24 specific 3'- flanking primer together with a vector specific primer. D2S44 inserts of various lengths were sequenced using the Amersham kit for cycle sequencing with fluorescent labelled primers. Automated sequencing was performed on an ALF DNA Sequencer.

\section{MVR-PCR}

MVR-PCR analysis of the D2S44 sequence in pYNH24 was done directly on plasmid DNA. Forty-eight genomic D2S44 alleles, with sizes from $1.45 \mathrm{kbp}$ to $6.95 \mathrm{kbp}$ when cleaved with HinfI, were amplified and separated by agarose-gel electrophoresis as previously described. ${ }^{15}$ Gel-slices with the PCR products were cut out, crushed and the DNA eluted into $100 \mu l$ of water. Five $\mu l$ of each of the DNA eluates was used for MVR analysis with the repeat specific primers \#1, \#2 and \#3 (Table1) in three separate reactions. The MVR-PCR reactions were set up with $25 \mathrm{pmol}$ of the universal primer together with $20 \mathrm{pmol}$ of one of the repeat-specific primers and $100 \mathrm{pmol}$ of the TAG-primer. The amplification mixture and amplification programme were in essence as earlier described. ${ }^{15}$ The MVR fragments were analysed in a $3 \%$ Hydrolink gel (FMC) on an ALF DNA Sequencer. Long D2S44 alleles were analysed from both the $3^{\prime}-$ and the 5'directions.

\section{Expected number of repeated core sequences}

To estimate the expected number of repeated core sequences the 49 alleles were analysed by Southern blotting after

Table 1 Primers used in MVR-PCR analysis of D2S44 alleles. The primer sequences are derived from sequence analysis of a $2.25 \mathrm{Kbp}$ HinfI D2S44 allele and from the pYNH24 plasmid. ${ }^{15,17}$ The TAG-sequence is virtually the same as used by Armour et al. ${ }^{7} 5^{\prime}$ - and $3^{\prime}$-orientations refer to the G-rich strand of the pUC18 D2S44 sequence ${ }^{15}$

\begin{tabular}{|c|c|}
\hline TAG-tail and primer & 5' TCATGCGTCCATGTTATTGA 3' \\
\hline \multicolumn{2}{|l|}{$5^{\prime}$ specific primers } \\
\hline Universal primer Cy5 labelled & 5' GGAAATAGTAGGAGGATGTTCA 3' \\
\hline Repeat primer \#1 & TAG $+\quad 5^{\prime}$ TGCTCCTGAACACCCCC $3^{\prime}$ \\
\hline Repeat primer \#2 & 5' CCCACTGCTCCTGAACAA 3' \\
\hline Repeat primer \#3 & 5' GCTCCTGAACACCCCAC 3' \\
\hline \multicolumn{2}{|l|}{ 3' specific primers } \\
\hline Universal primer Cy5 labelled & 5' AGCTGCTCCTAAACAACC 3' \\
\hline Repeat primer \#1 & TAG+ $\quad$ 5' GGGAAGTACAGTGGGGG 3' \\
\hline Repeat primer \#2 & 5' GGGAAGTACAGTGGGGTT 3' \\
\hline Repeat primer \#3 & 5' GGAAGTACAGTGGGGTG 3' \\
\hline
\end{tabular}


cleavage with both HinfI and HaeIII. The fragment sizes were determined by image processing. ${ }^{19}$ The mean length difference between HinfI and HaeIII fragments was $1150 \mathrm{bp}$. The number of bases in the sequenced $2.25 \mathrm{kbp}$ allele from the HaeIII site to the beginning of the repeats at the $5^{\prime}$ end was $86 \mathrm{bp}$, and $495 \mathrm{bp}$ at the 3 ' end. Assuming identity in flanking sequences, since no HinfI or HaeIII restriction site polymorphism was found, we estimated the number of repeats by subtracting $1731 \mathrm{bp}$ (sum of $1150+86+495 \mathrm{bp}$ ) from the HinfI fragment length and divided the result by 31 , the number of nucleotides in the published core sequence. ${ }^{1}$ The HinfI-size of the pYNH24 allele was obtained from Southern blot analysis of the YNH24 $40 \mathrm{kbp}$ cosmid DNA.

\section{Allele Alignment}

To align alleles objectively we used the 'Automatic multiple sequence alignment' in the DNASIS software (Hitachi Software Engineering Europe SA) originally designed for the comparison of primary DNA sequences. As we had four different repeats, \#1, \#2, \#3 and an unknown $\mathrm{x}$ (see results), DNASIS could easily be used by renaming the repeats to A, $\mathrm{C}, \mathrm{G}$ and $\mathrm{T}$ respectively. In some of the very long alleles we were not able to analyse the whole repeat array as estimated from the Hinf I fragment length. The number of repeats missed in these alleles were called $\mathrm{N}$, assuming that any of the ACGTs could be present. Due to limitations in the DNASIS program we could only align 30 of the alleles. These alleles were picked at random and alignment was done using the default parameters given in the software. Repeat motif similarities were also found by visual inspection and the repeat order motifs shared between the 49 alleles are illustrated with colours.

\section{Results and Discussion}

\section{Nucleotide Variation in the Core Sequence}

Clones with inserts of various lengths were sequenced and combined to give the sequence of the complete repeat array and $525 \mathrm{bp}$ of the 3 '- flanking sequence in the $2.25 \mathrm{kbp}$ allele (EMBL accession \# AJ001534). The first 22 bases at the $5^{\prime}$ - end of the core sequence is identical in all repeats, 5'- CAGGAGCAGTGGGAAGTACAGT... - -3'. However, at the 3'-end small differences in the number of Gs and Ts were found: ...(G) $)_{5}$ TGTT (repeat \#1), ...(G) $)_{4}$ TTGTT (repeat \#2) and ...(G) $)_{4}$ TGTT (repeat \#3) (Table 2). Repeat \#2 is identical to the sequence given by Nakamura et al. ${ }^{1}$ All directions given refer to the G-rich strand in the pUC18 D2S44 sequence. $^{15}$

Table 2 Core sequence variation in a $2.25 \mathrm{kbp}$ HinfI D2S44 allele

\begin{tabular}{ll}
\hline Repeat \#1 & $5^{\prime}-\ldots . .$. TGGGGGTGTT 3' \\
Repeat \#2 & $5^{\prime}-\ldots .$. TGGGGTTGTT 3 \\
Repeat \#3 & $5^{\prime}$-......TGGGGTGTT 3' \\
\hline
\end{tabular}

\section{MVR-PCR Analysis}

The specificity of the three repeat primers was confirmed by comparing the MVR results of the D2S44 sequence in pYNH24 with the nucleotide sequence kindly provided by Kishida $e t a l^{17}$ and the MVR results of the $2.25 \mathrm{kbp}$ allele with the sequence given in accession \# AJ001534. The MVR results were in complete agreement with the respective nucleotide sequences.

MVR-analysis of D2S44 alleles with the three repeat primers, a so-called three-state MVR-PCR, revealed a fourth unknown core sequence here called $\mathrm{x}$. The MVR analysis thus gave results comparable to a four-state MVR. However, the $\mathrm{x}$ variant has not been sequenced and we can therefore not be sure that this repeat is always the same. When analysing the alleles on the ALF sequencer we were able to read 35-45 repeats. Applying MVR analysis from both directions, 3'- and 5'MVR, made it possible to analyse up to 90 repeats $(2700 \mathrm{bp})$. The number of repeats in each allele agreed very well with the estimated number, differing by only one or two (Figure 1). This small discrepancy in number is most probably due to the limited resolution in restriction fragment length analysis, ${ }^{18}$ or to false annealing to degenerate repeats at the 5 ' end of the alleles. When analysing long alleles containing more than 90 repeats we were not always able to read through the whole repeat array. In these cases we have left a gap in the repeat arrays which corresponds to the number of repeats not identified. The very short allele of $1452 \mathrm{bp}$, found only once in a mother-child pair, seems to be the product of a mutation event since subtraction of the 1750 flanking bases would give a negative value. This allele was primarily only detected by PCR amplification, extended exposure after hybridisation revealed the $\operatorname{HinfI}$ fragment. In the very long 6953 bp allele, with 168 estimated repeats, we were able to analyse 110 repeats when combining the $663^{\prime}$ repeats and the $445^{\prime}$ repeats. In this allele we found a 24 repeat long overlap (green motif, Figure 1) between the 3 '- and 5'- repeat arrays. If this array is present only once in the allele the total repeat array would contain only 86 repeats, ie 58 repeats less than the estimated number. Most probably there is a gap of 58 repeats since neither the difference between the HinfI and HaeIII sizes nor the amplified allele length deviate from expected values.

\section{Repeat Order Motifs in Different Alleles}

The result from the alignment of 30 randomly selected alleles is shown as a phylogenetic tree in Figure 2. Three 


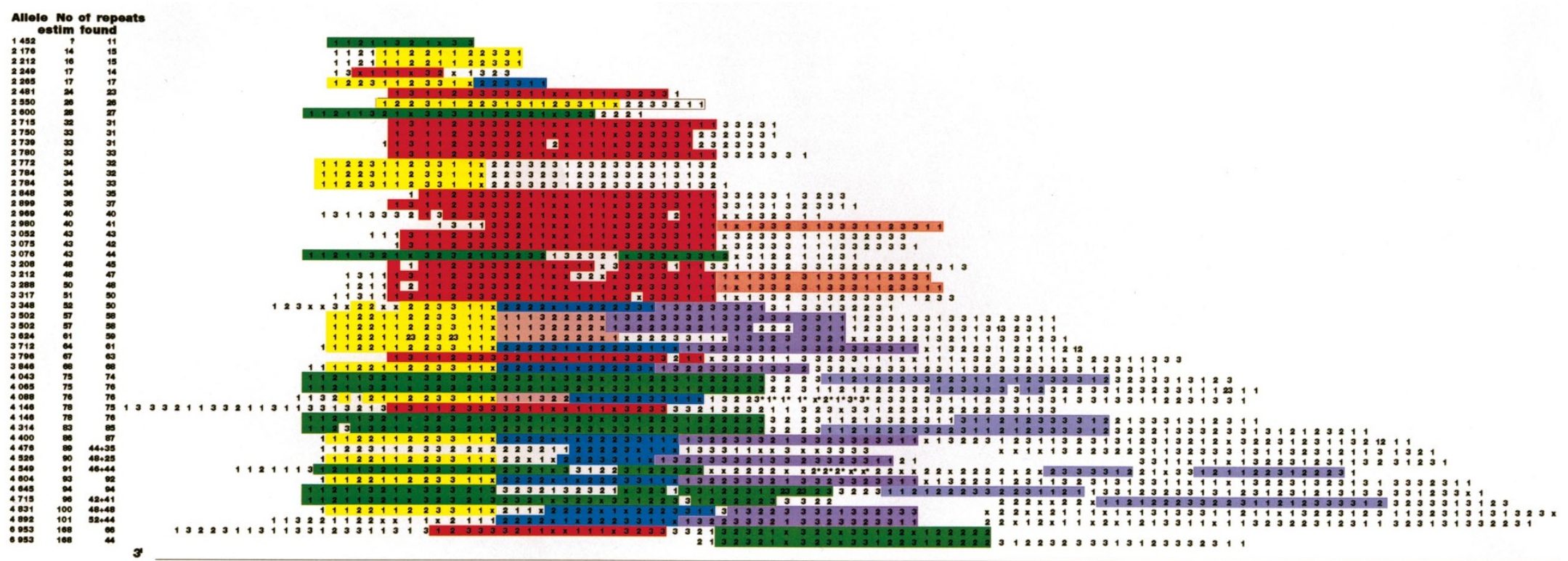

Figure 1 MVR-PCR repeat orders in D2S44 alleles are given by the repeat variant numbers 1,2,3 and an unknown variant called $x$. Some uncertain variants are indicated by a double designation or an asterisk. The red, green and yellow colours are used to denote highly conserved repeat order motifs present in different alleles. Blue, lilac, light-brown and light-blue colours indicate some less conserved motifs. Allele sizes as well as the number of expected and found repeats are shown to the left. The repeat order of pYNH24 is shown in a box (allele 2550) and the allele sequenced is the $2249 \mathrm{bp}$ allele. 


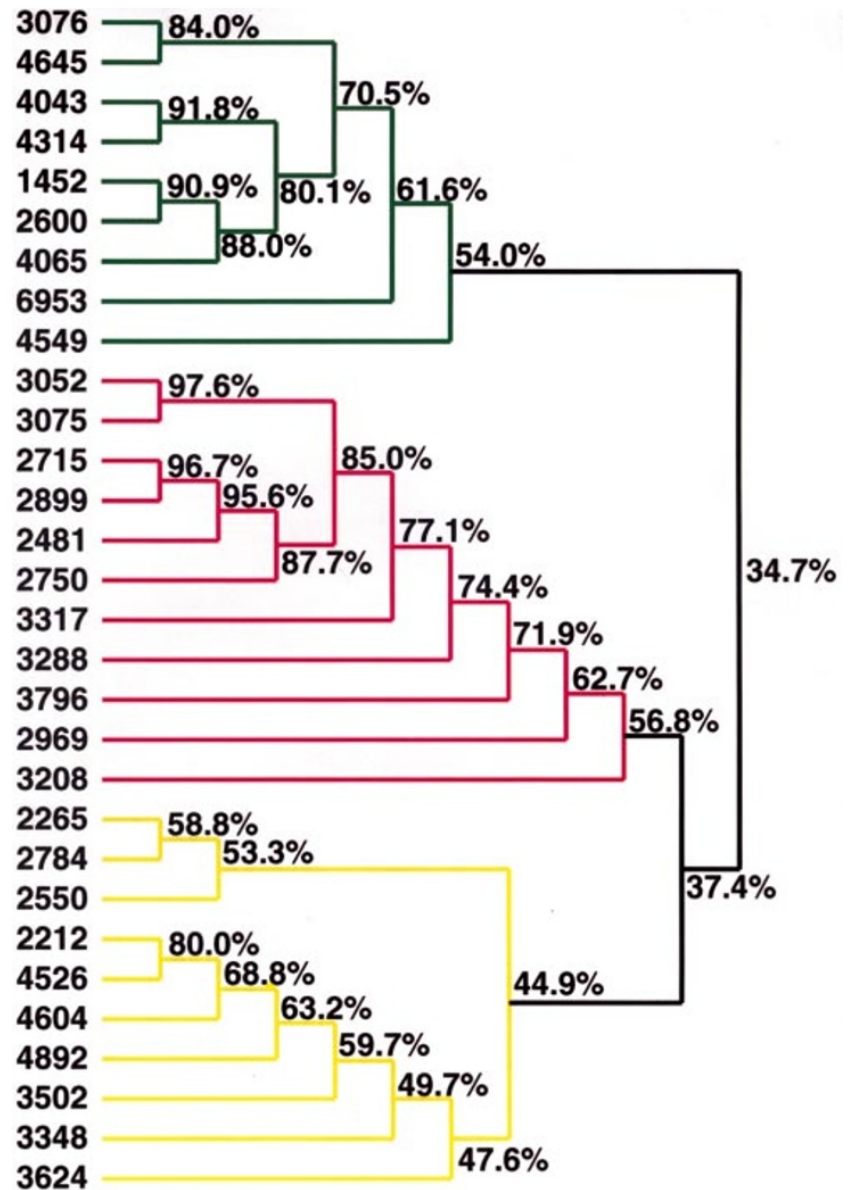

Figure 2 Repeat order similarities between 30 randomly selected D2S44 alleles given as a phylogenetic tree. The alleles were aligned progressively applying the UPGMA method ${ }^{19}$ used in the DNASIS software and the calculated match percentages are given at each branch point. Three major allelic clusters are shown by the colours green, red and yellow with the individual allele sizes in base pairs to the left.

clusters (green, red and yellow) with closely related alleles were found. In two of these clusters, the green and the red, the alleles have more than $50 \%$ similar repeat orders. The third yellow cluster contains alleles with a more than a $40 \%$ match. The alleles in the green cluster share the motif shown by the green colour in Figure 1. The alleles in the red group share the red motif, and the in the yellow group the alleles share the more complex yellow-blue-lilac motifs. The repeat order in the green alleles differs by $65 \%$ from the repeat orders in the other two clusters, and the repeat orders in the red and the yellow clusters differ from each other by $63 \%$. Similar clusters were found when aligning different combinations of the 49 alleles analysed.
D2S44 shows the same kind of a highly polarised variation found in most other minisatellites. ${ }^{5-7,10}$ The conserved repeat order motifs were always found at the $3^{\prime}$ end of the alleles, whilst the 5' end was highly variable. In contrast to all other minisatellites studied $^{5-11}$ the repeat order was highly correlated to the allele length. More than half of the allele length in the red alleles consists of the conserved red motif. Slightly less than half of the green alleles consists of the green motif and in the yellow alleles less than one fourth of the total length is represented by the yellow motif. However, together with the blue and lilac motifs about half of the yellow alleles consists of a conserved motif. The red motif (up to 26 repeats long) and the yellow motif (13 repeats) are the most conserved ones, followed by the green motif (up to 37 repeats), whilst the shorter blue and lilac motifs (15 and 19 repeats respectively) vary more and are often combined with the yellow motif. In long alleles a few less conserved motifs were also present at the 5 ' end (orange and light blue). Small (up to $2.55 \mathrm{kbp}$ ) and very large alleles $(6.95 \mathrm{kbp})$, found at low frequencies in the population, seem to be products of mutation events since they consist of only a part of a conserved motif or a fusion between, or duplication of, different motifs. Further aspects of the evolution of D2S44 alleles will be revealed by studies of de novo mutations (manuscript in preparation).

\section{Repeat Order Motifs in Comparison with the Bimodal Fragment Size Distribution}

The MVR-PCR results of D2S44 alleles belonging to the two groups of fragment sizes in the bimodal frequency distribution show that the alleles in the two groups must have arisen from different ancestor alleles. The differences found between the red, green and yellow allele clusters cannot have evolved from an early deletion or duplication in a common ancestor allele. Instead, the polymorphism of the D2S44 locus most probably descends from small alterations in the length of three different ancestor alleles of which one (red motif) has given rise to the alleles in the group around $2.8 \mathrm{kbp}$ and two others (green and yellow-blue-lilac) have evolved into the alleles in the group around $4.1 \mathrm{kbp}$ (Figure 3). These ancestor alleles might be older than the fusion of the two primate chromosomes that gave chromosome number 2 of the modern human., This theory could be confirmed by MVR-PCR analysis of D2S44 alleles in different human populations in particular of African origin and also by analysis of 
4 Yunis JJ, Prakash O. The origin of man: A chromosomal pictorial legacy. Science 1982; 215: 1525-1530.

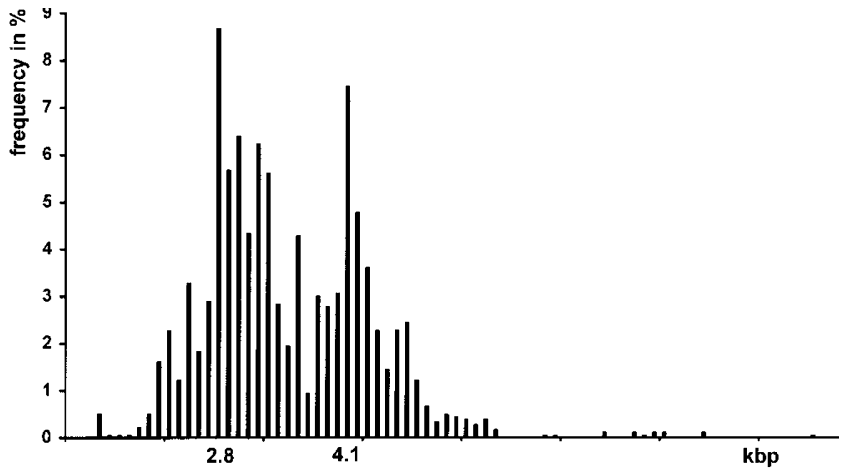

Figure 3 The frequency distribution of D2S44 HinfI fragments is divided into two groups with the most frequent fragment sizes around $2.8 \mathrm{kbp}$ and $4.1 \mathrm{kbp}$ respectively. The fragment sizes found in 1000 unrelated Swedish Caucasian individuals are binned into $100 \mathrm{bp}$ wide classes (author's unpublished data).

D2S44 in higher primates. Such studies are in progress and will be presented separately.

\section{Acknowledgements}

This work was funded by grants from the National Board of Forensic Medicine, Sweden and from the Forensic Science Center, University of Linköping. We want to thank Marko Marttila who did the cloning and sequencing of the $2.25 \mathrm{kbp}$ allele, Lisbeth Engdahl who did the MVR-PCR analysis and Birgitta Wahlgren who analysed the D2S44 alleles with HinfI and HaeIII for their skilful help. We also want to express our gratitude in particular to Sir AJ Jeffreys and Dr DL Neil at the Institute of Human Genetics, Leicester University, for valuable and inspiring discussions.

\section{References}

1 Nakamura Y, Leppert M, O'Connell $\mathrm{P}$ et al: Variable number of tandem repeat (VNTR) markers for human gene mapping. Science 1987; 235: 1616-1622.

2 Korenberg JR, Rasmussen IM, Chen X-N, Cohn DH: High resolution physical mapping of 8 polymorphic markers in the pericentromeric region of chromosome 2. Cytogenet Cell Genet 1993; 64: 87.

3 Ijdo JW, Baldani A, Ward DC, Reeders ST, Wells RA: Origin of human chromosome 2: An ancestral telomeretelomere fusion. Proc Natl Acad Sci USA 1991; 88: 9051-9055.
5 Jeffreys AJ, Neumann R, Wilson V: Repeat unit sequence variation in minisatellites: A novel source of DNA polymorphism for studying variation and mutation by single molecule analysis. Cell 1990; 60: 473-485.

6 Jeffreys AJ, MacLeod A, Tamaki K, Neil DL, Moncton DG: Minisatellite repeat coding as a digital approach to DNA typing. Nature 1991; 354: 204-209.

7 Armour JAL, Harris PC, Jeffreys AJ: Allelic diversity at minisatellite MS205 (D16S309): evidence for polarized variability. Hum Mol Genet 1993; 2: 1137-1145.

8 Armour JAL, Anttinen T, May CA et al: Minisatellite diversity supports a recent African origin for modern humans. Nat Genet 1996; 13: 154-160.

9 Buard J, Vergnaud G: Complex recombination events at the hyper mutable minisatellite CEB1 (D2S90). EMBO J 1994; 13: 3203-3210.

10 Neil DL, Jeffreys AJ: Digital DNA typing at a second hypervariable locus by minisatellite variant repeat mapping. Hum Mol Genet 1993; 2: 1129-1135.

11 Armour JAL, Crosier M, Jeffreys AJ: Distribution of tandem repeat polymorphism within minisatellite MS621 (D5S110). Ann Hum Genet 1996; 60: 11-20.

12 Jeffreys AJ, Allen MJ, Armour JAL et al: Mutation processes at human minisatellites. Electrophoresis 1995; 16: $1577-1585$.

13 Dubrova YE, Nesterov VN, Krouchinsky NG et al: Human minisatellite mutation rate after the Chernobyl accident. Nature 1996; 380: 683-686.

14 Appelgren $\mathrm{H}$, Cederberg H, Rannung U: Mutations at the human minisatellite MS32 integrated in yeast occur with high frequency in meiosis and involve complex recombination events. Mol Gen Genet 1997; 256: 7-17.

15 Holmlund G, Lindblom B: Flanking region sequences and internal repeat structure of the pYNH24 (D2S44) $2 \mathrm{kbp}$ insert analysed by polymerase chain reaction and partial digestion with RsaI. Electrophoresis 1995; 16: 1881-1885.

16 Budowle B, Monson KL: Greater differences in forensic DNA profile frequencies estimated from racial groups than from ethnic subgroups. Clin Chim Acta 1994; 228: 3-18.

17 Kishida T, Tamaki Y, Kuroki K, Fukuda M, Wang W: PCR amplification of D2S44 (YNH24) alleles. Japanese Journal of Legal Medicine (Nippon Hoglaku Zasshi) 1995; 49: 299-303.

18 Holmlund G, Karlberg K, Gustavsson B, Lindblom B: Calculation of restriction fragment lengths by image processing. Electrophoresis 1992; 13: 407-410.

19 Sneath PHA, Sokal RR: Numerical Taxonomy. The principles and practice of numerical classification. In: Kennedy D, Park RB (ed.): A Series of Books in Biology. WH Freeman: San Francisco, 1973. 